\title{
Desenvolvimento e implantação de Terapia Assistida por Animais em hospital universitário
}

\author{
Development and implementation of Animals-Assisted Therapy in a universitary hospital
}

Desarrollo y aplicación de la Terapia Asistida por Animales en el hospital universitario

\author{
Cassia Tiemi Kobayashi', Sílvia Tiemi Ushiyama", Flávio Trevisan Fakih', \\ Roseli A. M. Robles', leda Aparecida Carneiro', Maria Isabel Sampaio Carmagnani' \\ 'Universidade Federal de São Paulo. Hospital São Paulo, Departamento de Enfermagem. São Paulo, SP \\ "Centro Universitário São Camilo. São Paulo, SP
}

Submissão: $30 / 07 / 2008$

Aprovação: 12/06/2009

\section{RESUMO}

O presente relato refere-se à experiência da Diretoria de Enfermagem do Hospital São Paulo da Universidade Federal de São Paulo no desenvolvimento e implantação da Terapia Assistida por Animais, como um de seus projetos de humanização hospitalar: "Projeto Amicão". Objetivando proporcionar aos pacientes uma experiência positiva Que difere da rotina do ambiente hospitalar, algumas unidades do Hospital São Paulo receberam a visita de um animal para sessões de Terapia Assistida por Animais. Os resultados alcançados entre pacientes, acompanhantes e profissionais da saúde foram positivos, além de despertar a atenção e o interesse de outras instituições de saúde e da mídia. Ficou, assim, evidenciada a importância de se relatar a experiência do "Projeto Amicão" no ambiente hospitalar.

Descritores: Terapias complementares; Enfermagem; Humanização da assistência.

\begin{abstract}
This report refers to the experience of the Board of Nursing of Hospital São Paulo, Universidade de São Paulo, in the development and implantation of Animal-Assisted Therapy, as one of its projects humanization of hospital: "Projeto Amicão". Aiming to offer patients a positive experience that differs from the routine of the hospital environment, some units of the Hospiral São Paulo received the visit of an animal for sessions of Animal-Assiste Therapy. The results achieved among patients and its companions and health professionals were positive, in addition to arouse the attention and the interest of other health institutions and the media. It is thus evidenced the importance of reporting the experience of "Projeto Amicão" in the hospital.
\end{abstract}

Descriptors: Complementary therapies; Nursing; Humanization of assistance.

\section{RESUMEN}

Este informe se refiere a la experiencia de la Junta de Enfermería, Hospital de Sao Paulo (HSP), Universidad Federal de Sao Paulo en el desarrollo y el despliegue de la Terapia Asistida por Animales (TAA), como uno de sus proyectos de humanización del hospital: "Proyecto Amicão". Con la finalidad de ofrecer los pacientes una experiencia positiva que difiere de la rutina del entorno hospitalario, algunas unidades de lo HSP recibió la visita de un animal para los períodos de sesiones de TAA. Los logros entre los pacientes, sus acompañantes y profesionales de la salud fueron positivos, y despertó la atención y el interés de otras instituciones de salud y de los medios de comunicación. Es así de relieve la importancia de comunicar la experiencia del "Proyecto Amicão" en el hospital.

Descriptores: Terapia s complementarias; Enfermería; Humanización de la asistencia. 


\section{INTRODUÇÃO}

A equipe de saúde tem um importante papel no processo de hospitalização sendo capaz de desenvolver e empregar estratégias para torná-la menos traumática. Beneficiando pacientes, acompanhantes e a própria equipe de saúde, o desenvolvimento de um plano de cuidado individualizado e humano também pode proporcionar momentos de alegria e descontração a fim de promover o paradigma orientado pela saúde e não pela doença ${ }^{(1)}$. Amparado por tal filosofia, o Programa Nacional da Assistência Hospitalar (PNHAH), instituído há 7 anos pelo Ministério da Saúde, objetiva humanizar a assistência hospitalar a partir de intervenções institucionais e construir uma cultura de atendimento à saúde da população pautada pelo respeito à vida humana. Para tanto, os valores Que norteiam esta política são a autonomia e o protagonismo dos sujeitos, a co-responsabilidade entre eles e o estabelecimento de vínculos solidários ${ }^{(2)}$.

Neste sentido, instituições de saúde do país desenvolveram formas alternativas de humanizar a assistência ao paciente hospitalizado e dentre as atividades mais inovadoras, destacamos a Terapia Assistida por Animais (TAA) $)^{(1,3-6)}$. A TAA é uma intervenção direcionada, individualizada e com critérios específicos em Que o animal é parte integrante do processo do tratamento. Esta intervenção deve ser aplicada e supervisionada por profissionais da saúde, devidamente habilitados, sendo todo o processo documentado e avaliado periodicamente, objetivando promover a melhora da função física, social, emocional e/ou cognitiva dos pacientes ${ }^{(4)}$.

Historicamente a TAA foi utilizada de forma pioneira e intuitiva em 1792 no tratamento de doentes mentais. A equoterapia, uma modalidade da TAA, teve seus primeiros relatos como tratamento médico no século XVIII, com o objetivo de melhorar o controle postural, a coordenação e o equilíbrio de pacientes com distúrbios $\operatorname{articulares}^{(7,8)}$.

Nos anos 60, o psiquiatra Boris Levinson desenvolveu a Psicoterapia Facilitada por Animais, utilizada no tratamento de transtornos de comportamento, déficit de atenção e problemas de comunicação em crianças $^{(8)}$.

Em decorrência dos resultados, nas últimas décadas a TAA tem sido aplicada em diferentes programas Que auxiliam a recuperação da saúde mental, obtendo melhora na comunicação, auto-estima e capacidade para assumir responsabilidades; nas interações sociais e também reduzindo a violência em pacientes e presidiários ${ }^{(4,9)}$. No Brasil, a psieuiatra Nise da Silveira encontrou na TAA uma forma de tratamento para a esquizofrenia. Entre outras atividades, gatos foram utilizados para o tratamento de doentes com distúrbios mentais $^{(4)}$. Em São Paulo, a médica veterinária e psicóloga Hannelore Fuchs coordena o projeto Pet Smile há dez anos, onde voluntários levam animais para interagir com crianças em hospitais ${ }^{(9)}$.

Em 2000, foi fundada a Organização Brasileira de Interação Homem-Animal Cão Coração, Que promove o Projeto Cão do Idoso. Tal atividade é aplicada em casas de repouso ou abrigos com a finalidade de melhorar a Qualidade de vida, o bem estar, integridade e respeito ao idoso ${ }^{(4-8)}$.

Em 2003, pesquisadores da Faculdade de Medicina Veterinária em parceria com a Faculdade de Odontologia da Universidade Estadual de São Paulo de Araçatuba, iniciaram o projeto "Cão Cidadão UNESP", Que investiga as reações provocadas pelos animais nas crianças com necessidades especiais, como paralisia cerebral, portadores de Síndrome de Down entre outros comprometimentos mentais. Os resultados foram satisfatórios e segundo o estudo, os pacientes apresentaram melhora no comportamento e colaboraram no atendimento dentário ${ }^{(7,8)}$.

Conduzido pela Faculdade Médica Veterinária e Zootecnia da Universidade de São Paulo, em Pirassununga, o projeto "Dr. Escargot" vem tentando provar Que os animais podem ser integrados ao meio escolar e hospitalar para proporcionar aprimoramento ético, moral, cidadania e Qualidade de vida às crianças e idosos ${ }^{(8)}$.

A Fundação Selma, em São Paulo, utiliza a equoterapia como alternativa para pacientes em reabilitação física e educação. Cães, ratos, coelhos, porQuinhos da índia e algumas aves têm auxiliado no trabalho com crianças e adolescentes no tratamento de problemas com linguagem, percepção corporal e controle da ansiedade ${ }^{(8-10)}$.

O médico geriatra Renato Maia, diretor do Centro de Medicina do Idoso do Hospital Universitário de Brasília e a veterinária Esther Odenthal, iniciaram um projeto piloto Que se tornou uma linha de pesquisa científica na Universidade de Brasília. A experiência envolveu pacientes do Centro de Referência para portadores de Doença de Alzheimer, a equipe de médicos e veterinários e dois cães. Os efeitos da terapia mediada por animais vão além da avaliação da memória dos pacientes, os Quais foram estimulados a memorizar os nomes e as cores dos animais. O estudo concluiu Que as sessões de TAA promoveram a melhora do humor e a recuperação de lembranças e acontecimentos recentes ${ }^{(10)}$.

Especialistas têm considerado Que a utilização do estímulo sensorial do tato com a presença e interação dos animais, pode recuperar a auto-estima e a sensibilidade além da reintegração à sociedade por meio da melhora do contato social Que o animal permite $^{(11,12)}$. A TAA traz consigo um aspecto importante de humanização, pois pode descontrair o clima tenso do ambiente hospitalar, melhorar as relações interpessoais e facilitar a comunicação entre pacientes e equipe de saúde. É indicada para auxiliar nas diversas situações clínicas, pois proporciona benefícios emocionais e espirituais para pacientes ${ }^{(9)}$.

Destacam-se ainda os benefícios específicos obtidos como aperfeiçoar as habilidades motoras finas; o equilíbrio de sustentarse; melhorar adesão ao tratamento; aumentar a interação verbal entre os membros do grupo; melhorar habilidades de atenção; desenvolver recreações e lazer; aumentar a auto-estima; reduzir a ansiedade; a solidão; aperfeiçoar o conhecimento dos conceitos de tamanho e cor; melhorar a interação com a equipe de saúde e a motivação para o envolvimento em atividades em grupo ${ }^{(1-16)}$.

A TAA pode ser aplicada em várias faixas etárias e em diferentes locais, tais como: hospitais, ambulatórios, casas de repouso, escolas, clínicas de fisioterapia e de reabilitação. São utilizados todos os tipos de animais Que possam entrar em contato com os humanos sem proporcionar-lhes perigo ${ }^{(10,15)}$.

O cão é o principal animal, pois apresenta uma natural afeição pelas pessoas, é adestrado facilmente, cria respostas positivas ao toque e possui uma grande aceitação por parte das pessoas. Estudos demonstram Que animais Que podem ser tocados resulta numa terapia mais efetiva ${ }^{(19,20)}$. Relatos referentes a algumas patologias mostram Que esta interação cão-paciente melhora o padrão cardiovascular, diminuindo a pressão arterial e os níveis de colesterol. Tal interação também produz o aumento da concentração plasmática 
de â-endorfinas, ocitocinas, prolactina, dopamina e diminui a concentração plasmática de cortisol, substâncias Que atuam positivamente no estado de ansiedade ${ }^{(3-20)}$.

Investigações têm demonstrado Que as sessões de TAA com cães e crianças hospitalizadas facilitaram a socialização, distração durante procedimentos dolorosos, companhia e lembranças de casa durante o processo de hospitalização ${ }^{(9,11,13,16-20)}$.

Apesar do reconhecido benefício bio-psico-social da TAA, os principais inconvenientes estão relacionados a mordidas de animais, alergias e zoonoses. É importante ressaltar Que estudos sobre infecção hospitalar mostraram ser mais comum um visitante humano transmitir infecções aos pacientes do Que os animais, Quando devidamente limpos e imunizados ${ }^{(21,22)}$. Ademais, estudos realizados após cinco anos de TAA em ambiente hospitalar, concluíram Que o número de infecções não alterou durante o período Que os animais estiveram presentes ${ }^{(23)}$.

O Hospital São Paulo (HSP) da Universidade Federal de São Paulo (UNIFESP) vem desenvolvendo um projeto de humanização hospitalar denominado Amicão ${ }^{(24)}$. Objetivando proporcionar aos pacientes uma experiência positiva Que difere da rotina do ambiente hospitalar, algumas unidades do HSP estão recebendo a visita semanal de uma voluntária e seu cão. Considerando os importantes resultados alcançados e o grande interesse por parte de profissionais desta e de outras instituições e até dos meios de comunicação, interessados em conhecer e divulgar esse projeto, concluímos ser necessário relatar à comunidade científica a experiência do Projeto Amicão.

\section{DESENVOLVIMENTO E IMPLANTAÇÃO DO PROJETO AMICÃO (PA)}

Criado em 2006, pelo Departamento de Pediatria e Diretoria de Enfermagem do Hospital São Paulo, o PA tem a finalidade de humanizar o ambiente hospitalar por meio da Terapia Assistida por Animais. Foi desenvolvido para ser implantado em algumas unidades de internação do HSP - hospital universitário de porte especial, situado na cidade de São Paulo, integrando o Programa de Humanização desta instituição - a descrição deste e de outros projetos podem ser encontrados no site oficial do Grupo de Trabalho de Humanização do HSP(24).

Para a seleção do animal, das unidades de internação e dos respectivos pacientes Que integraram o $\mathrm{PA}$, foram consideradas as recomendações, critérios e normas descritas a seguir:

a) Recomendações do Centers for Disease Control and Prevention (CDC) e do Healthcare Infection Control Practices Advisory Committee (HICPAC) ${ }^{(21,22)}$ :

Estas organizações americanas apresentam recomendações relacionadas à TAA, subdivididas em três partes: Quanto ao animal, ao paciente e à coordenação do programa de $\operatorname{TAA}^{(21,22,23)}$.

\section{Quanto ao animal:}

- Deve realizar avaliação veterinária periódica e apresentar certificado de saúde;

- Deve realizar tratamento antiparasitário intestinal periodicamente;

- Não ser portador de Salmonella SP, Campylobacter SP ou Giárdia intestinal, ou até Que estejam tratados e tenham teste negativo para as mesmas;

- Ser selecionado e treinado por profissionais;

- Deve devem tomar banho previamente às visitas (menos de 24 horas);

- Deve ter tosas periódicas (conforme o tipo e a raça do animal);

- Não pode ter contato com outros animais de rua;

- Deve ter a avaliação, a aprovação e a autorização da Comissão de Infecção Hospitalar.

\section{Quanto ao paciente:}

- Deve concordar em receber a visita do animal (os menores de idade devem ter a autorização prévia dos pais ou responsável);

- Não é recomendada a participação de pacientes Que apresentarem fobia por animais, além dos Que forem imunocomprometidos, esplenectomizados, neutropênicos, ou apresentarem alergias e problemas respiratórios;

- Evitar maltratar o animal, induzindo-o a uma resposta violenta;

- Realizar a higiene das mãos após o contato com os animais;

- Evitar que o animal lamba pele, feridas ou dispositivos.

- Evitar o contato com saliva, urina e fezes dos animais;

- Informar Qualquer incidente com o animal (como mordidas, arranhões, ou alterações de comportamento do animal) à coordenação do programa e à Comissão de Controle de Infecção da instituição. A área do arranhão, mordida ou de Qualeuer outro acidente deverá ser prontamente higienizada, conforme orientação da equipe de saúde do local.

\section{Quanto à Coordenação do Programa e Equipe de Saúde:}

- É recomendável a concordância prévia do corpo clínico responsável pela unidade hospitalar onde se pretende implantar a TAA;

- Não se recomendam visitas em unidades de terapia intensiva, no entanto, alguns pacientes crônicos e conscientes, além da equipe de saúde, poderão beneficiar-se da TAA;

- Limitar o acesso dos animais nas áreas de preparação de alimentos e medicação, lavanderia, central de esterilização e desinfecção, sala de cirurgia e de isolamento;

- As visitas deverão ocorrer junto ao treinador e a um profissional da equipe de saúde.

\section{b) Seleção do Animal:}

Os aspectos mais importantes Que um animal deve apresentar para integrar o PA, segundo critérios do CDC e HIPAC, são: ter comportamento amigável com estranhos e estar habituado com sessões de TAA em ambiente hospitalar ${ }^{(21-25)}$. Considerando tais aspectos, executou-se um projeto piloto Que utilizando um cão da raça Golden Retriever, de dois anos de idade, chamado Joe.

Previamente ao início das visitas, o PA foi submetido à avaliação e aprovação da Comissão de Controle de Infecção Hospitalar (CCIH) do HSP. Esta comissão exigiu Que o PA atendesse as seguintes rotinas: apresentar, semestralmente, atestado de saúde e vacina do cão, inclusive contra Giárdia. Além disso, recomendaram que se utilizasse elevador privativo e Que se evitasse circular por áreas do hospital Que não as selecionadas para as visitas.

Além de atender aos critérios descritos acima, de já ter participado de sessões de TAA em dois asilos, de freqüentar sessões periódicas de adestramento e de obedecer prontamente aos 
comandos de sua proprietária, o comportamento calmo e o temperamento extremamente dócil e amigável deste cão facilitaram sua aprovação para integrar definitivamente o PA. As figuras 1, 2 e 3 exemplificam a interação cão-paciente no PA.

\section{c) Seleção das unidades hospitalares:}

As unidades hospitalares integrantes do PA foram selecionadas considerando os critérios do CDC e HIPAC, a saber: tipo de paciente e Quadro clínico, estrutura física da unidade e receptividade do corpo clínico em relação ao $\mathrm{PA}^{(21-23)}$.

Foram escolhidas as unidades de Clínica Pediátrica, Cirurgia Pediátrica, Psiquiatria e Setor Pediátrico do Hospital-dia. Essas unidades foram submetidas à aprovação da CCIH do HSP e, então, solicitaram-se as autorizações aos médicos responsáveis por essas unidades. Após a obtenção dessas autorizações, o Diretor Superintende e Diretor Clínico desta instituição aprovaram e consentiram a presença do animal nas dependências do hospital, conforme as exigências apontadas.

\section{d) Visitas aos pacientes:}

Para Que as sessões de TAA do PA pudessem apresentar resultados positivos, tanto para pacientes e familiares Quanto à equipe de saúde, foi necessário incorporá-las às rotinas da unidade selecionada, de modo que período entre as visitas do animal fosse regular e não muito longo ${ }^{(9,13,18)}$. Assim, desde o início do PA, há dois anos, loe visita as unidades uma vez por semana, em dias e períodos fixos, permanecendo em contato direto com os pacientes e profissionais durante o período da tarde.

Acompanhado por sua proprietária e voluntária do PA, Sra. Lucy da Silva, pela assistente social da Pediatria e por um profissional da Diretoria de Enfermagem do HSP, Joe percorre as unidades hospitalares integrantes do PA, munido de um crachá de identificação institucional (fixado em sua coleira). Durante as visitas, habitualmente, ele se comporta da seguinte maneira: não aceita alimentos ou bebidas durante as sessões, não faz necessidades no ambiente hospitalar e adjacências e obedece aos comandos da proprietária, Que por sua vez atende as solicitações dos pacientes, familiares, acompanhantes e profissionais.

$\mathrm{Na}$ unidade de Pediatria Clínica, Joe deita-se, brinca pelo saguão de entrada e permanece por cerca de 40 minutos. As crianças que podem se locomover, vão ao seu encontro para conversar, acariciar, pentear, brincar e tirar fotos com ele. Na Unidade de Cirurgia Pediátrica, onde se encontram crianças acamadas, após a prévia autorização do familiar ou acompanhante, além da própria criança, loe senta-se em uma poltrona ao lado do leito e permanece por cerca de 5 minutos. Na Unidade de Psiquiatria, o cão deita-se na área de convivência da unidade e recebe a visita dos pacientes que brincam, conversam e acariciam o animal por cerca de 40 minutos. A mesma rotina é realizada junto às crianças Que aguardam procedimento no
Setor do Hospital dia, anexo à unidade de Cirurgia Pediátrica.

\section{CONSIDERAÇÕES FINAIS}

Em dois anos de sessões de TAA do Projeto Amicão, há relatos de pacientes e profissionais favoráveis à continuação da terapia. Anotações em registros de enfermagem, prontuários médicos e avaliações de estágio forneceram resultados expressivos em relação à presença de Joe nas unidades visitadas. O feedback do PA com profissionais e pacientes desta Instituição demonstrou, de fato, sua importância no processo de humanização da assistência.

As mães das crianças internadas na Pediatria referem Que as mesmas aguardam a chegada de loe com alegria e expectativa. A expressão de felicidade dos pacientes e profissionais desta unidade ao ver o loe foi sempre compensatória, pois segundo comentários durante as visitas as crianças param de chorar, os adultos ficam enternecidos e entusiasmados. Todos se juntam em volta ao cão e o grupo só se desfaz Quando ele vai embora.

Os residentes da Psiquiatria solicitaram, durante avaliações de estágio, o aumento do número de visitas do Joe, referindo que sua presença alegrava e diversificava o dia-a-dia dos pacientes e da unidade.

Os freqüentes convites de profissionais desta e outras instituições para implementação de TAA semelhante, devem-se aos resultados positivos obtidos com as visitas de loe, os Quais geraram grande popularidade do PA neste hospital e na mídia.

Desde o início das visitas, não houve incidentes como reação agressiva do cachorro ou o fato de algum paciente maltratá-lo. Aspectos como o temperamento amigável de Joe, a periodicidade de sua presença e a interação da proprietária do cão com os pacientes e familiares - sempre colaborativa e bem humorada foram aspectos fundamentais para o sucesso da TAA.

O Projeto Amicão mudou, de forma significativa, a rotina das Quartas-feiras do HSP. Joe não passa desapercebido nos corredores desta instituição. Com freeüência, transeuntes do hospital aproximamse do cão para conversar, acariciá-lo e tirar fotos com ele. Vale ressaltar Que ele também atrai a atenção dos profissionais de saúde, de todas as áreas, os Quais também interagem afetivamente com o animal.

Este projeto é hoje referência do processo de humanização da assistência no Hospital São Paulo. Após análise dos relatos nos últimos anos de TAA, ficou evidente a necessidade de se ampliar as sessões do PA para outras unidades e aumentar o número de visitas nas unidades atuais. No entanto, é fundamental a realização de mais estudos sobre a Terapia Assistida com Animais e seus benefícios para a saúde dos pacientes. Será necessário, portanto, estudar e verificar a reação positiva na diminuição dos sinais e sintomas dos pacientes assistidos por esta terapia, tais como: diminuição da pressão arterial e dor, melhoria da adesão ao tratamento além dos sintomas de ansiedade e depressão.

\section{REFERÊNCIAS}

I. Bussotti EA, Leão ER, Chimentão DMN, Silva CPR. Assistência individualizada: "Posso trazer meu cachorro?" Rev Esc Enferm USP. 2005; 39(2): 195-201.

2. Ministério da Saúde (BR). Política Nacional de Humanização [citado 15 jun 2008]. Disponível em: URL: www.saude.gov.br/ humanizasus

3. Jofre ML. Animal Assisted Therapy in health care facilities. Rev Chilena Infectol 2005; 22(3): 257-63.

4. Juliano RS, Fioravanti MCS, Paulo NM, Athayde IB. Terapia Assistida por Animais (TAA): uma prática multidisciplinar para o benefício da saúde humana. [citado 15 jun 2008]. Disponível em: URL: www.vet.ufg.br/ 
5. Zooterapia. Organização da Sociedade Civil de Interesse Público [citado 15 jun 2008]. Disponível em: URL: www.zooterapia. com.br/

6. Organização Brasileira de Interação Homem-Animal. [citado 15 jun 2008]. Disponível em: URL: www.projetocao.org.br

7. Oliva VNLS. A terapia assistida por animais: o papel do médico veterinário. Bol Inform ANCLIVEPA-SP 2008; 35. [citado 15 jun 2008]. Disponível em: URL: www.anclivepa-sp.org.br/ inicio.htm

8. Martins MF. Zooterapia ou terapia assistida por animais (TAA). Rev Nosso Clínico 2004; 40: 22-6.

9. Vaccari AMH, Almeida FA. A importância da visita de animais de estimação na recuperação de crianças hospitalizadas. Einstein 2007; 5(2): 1 II-6.

10. Centro de Medicina do Idoso do Hospital Universitário de Brasília Dosagem animal. [citado 15 jun 2008]. Disponível em: URL: www.kalunga.com.br/revista/revista_maio_05.asp

1 1. Delta Society. Standarts of Practice in Animals Assisted Activity and Theraphy. Washington: Renton; 1996.

12. San JoaQuín MPZ. Terapia assistida por animales de conpañia. Bienestar para el ser humano. Temas de Hoy 2002:143-9.

13. Kawakami CH, Nakano CK. Relato de experiência: Terapia Assistida por Animais (TAA) - mais um recurso na comunicação entre pacientes e enfermeiro. In: Anais do $8^{\circ}$ Simpósio Brasileiro de Comunicação em Enfermermagem. Maio 2002; São Paulo (SP), Brasil. Disponível em: http://www.proceedings.scielo.br/ scielo.php

14. Allen K. Dog ownwership and Control of borderline hypertension: A control randomized trial. Division of clinical Pharmacology Department of Medicine. Bufalo: Millard Fillmore Hospital; 2001 .

15. Fila D. The significance of companion animals to a geriatric vascular case study. Holistic Nurs Prac 1991; 5:2.
16. Friedman E, Katcher A, Lynch J, Thomas S. Animal companions and one-year survival of patients after discharge from a coronary care unit. Public Health Reports 1980; 95:307-312.

17. Katcher AH. Physiological and beyavioral responses to companion animals. Vet Clin North Am 1985; 26: 403-9.

18. Diniz M. O convívio com os animais traz alegria, bem estar e saúde. Conheça histórias de Quem se apoiou nos bichinhos para superar os mais diversos problemas [citado 15 jun 2008]. Disponível em: URL: www.projetocao.org.br

19. Kirwen S. "Animal - Assisted Theraphy as a modality within speech and language theraphy" in therapeutic interventions. Bellevue: Delta Society; 1996.

20. Sobo JE. Canine Visitation (pet) Theraphy - pilot data on decreases in child pain perception. I Holistic Nurs 2006; 24:5 I-7.

21. Guidelines for environmental Infection Control in Healthcare Facilities. Recommendations of CDC and the Healthcare Infection Control Pratics Advisory Committee (HICPAC). U.S. Department of Health and Human Service Centers for Disease Control and Prevention (CDC). Atlanta: Centers for Disease Control; 2003.

22. Guidelines for Environmental Infection Control in Health-Care Facilities. MMWR/CDC Recomm Reports 2003; 6/52(RRI0): I-42 [citado I 5 jun 2008]. Disponível em: URL: www.cdc.gov/ mmwR/preview/mmwrhtml/rr52 I Oal.htm

23. Khan MA, Farrag N. Animal-Assisted Activity and Infection Control implications in a Healthcare Setting. London: St Georges Hospital; 2000.

24. Hospital São Paulo. Projeto Humanização do Hospital São Paulo. [citado 15 jun 2008]. Disponível em: URL: www.unifesp.br/ spdm/hsp/humaniza/p03.htm

25. Havener L, Gentes L, Thaler B, Megel ME, Baun MM, Driscoll FA, et al. The effects of a companion animal on distress in children undergoing dental procedures. Iss Compr Pediatr Nurs 2001; 24(2): 137-52. 